\title{
Review \\ The importance of being a myoepithelial cell
}

\section{Marie-Ange Deugnier, Jérôme Teulière, Marisa M Faraldo, Jean Paul Thiery and Marina A Glukhova}

UMR 144 CNRS-Institut Curie, Section de Recherche, Paris, France

Correspondence: Marina A Glukhova, UMR 144 CNRS-Institut Curie, Section de Recherche, 26 rue d'Ulm, 75248 Paris, Cedex 05, France. Tel: +33 14234 6333; fax: +33142346349; e-mail: Marina.Glukhova@curie.fr

Received: 13 June 2002

Breast Cancer Res 2002, 4:224-230 (DOI 10.1186/bcr459)

Revisions requested: 16 July 2002

Revisions received: 18 July 2002

Accepted: 25 July 2002

Published: 19 August 2002

(C) 2002 BioMed Central Ltd

(Print ISSN 1465-5411; Online ISSN 1465-542X)

\begin{abstract}
The mammary myoepithelial cell was named the 'Cinderella of mammary cell biology' in light of the earlier focus on the luminal cell. Mammary myoepithelial cells have recently been described as 'natural tumour suppressors'. We now need to understand more about their origin and to reconsider their place in the complex process of mammary morphogenesis. In the present review, we discuss the lineage segregation of mammary myoepithelial cells and their functions in mammary gland development. These functions include their effects on luminal cell growth and differentiation, their key role in the establishment of the polarised mammary epithelial bilayer and the control of stromal invasion in breast cancer.
\end{abstract}

Keywords: breast cancer, cell differentiation, mammary gland, morphogenesis, myoepithelial cell

\section{Introduction}

The mammary gland consists of secretory alveoli connected by a system of branching ducts embedded in connective tissue. The epithelial cells that compose the gland are arranged in two layers, the luminal epithelial layer and the basal myoepithelial layer. The whole structure is surrounded by a basement membrane.

The myoepithelial layer is organised differently in the ducts and in the lobules. In the ducts, elongated myoepithelial cells form a more or less continuous layer and are in direct contact with the basement membrane, and hence with the stroma. The interaction between ductal luminal cells and the extracellular matrix $(E C M)$ is largely mediated by the myoepithelium, although some of the luminal cells in the mammary ducts may reach the basement membrane. Alveolar myoepithelial cells are of stellate shape and form a basket-like structure around the acini, resulting in the exposure of most of the basal surface of the luminal cell to the basement membrane.
Differentiated myoepithelial cells are highly contractile and their ultrastructure is reminiscent of that of smooth muscle cells. Myoepithelial cells contain large amounts of microfilaments, dense plaques (cell-matrix adherens junctions characteristic of smooth muscle cells) and smooth muscle-specific cytoskeletal and contractile proteins. They are true epithelial cells, however, because the major components of their intermediate filament system are the cytokeratins 5 and 14 ( $K 5$ and $\mathrm{K} 14$ ), because they form desmosomes, hemidesmosomes and cadherin-mediated cell-cell junctions, and because they are permanently separated from the connective tissue by the underlying basement membrane.

The contractile properties and the central role in milk ejection during lactation are the most studied aspects of mammary myoepithelial cell function. In addition, the tumour suppressor potential of mammary myoepithelial cells has recently received considerable attention. However, the role played by the myoepithelial cells in 
mammary development remains poorly investigated. The vast majority of studies devoted to various aspects of mammary gland development to date have focused on the mechanisms controlling luminal cell differentiation and growth, whereas basal myoepithelial cells have been largely neglected.

In the present review, we refer to the studies carried out in the mouse, the rat and the human. Although mammary gland development, function and pathology are obviously not identical in different mammalian species, the data obtained in rodent models, when considered at the cellular and molecular levels, provide essential information relevant to human breast disease.

\section{Differentiation of the mammary myoepithelial cell}

In the rat, for most of the embryonic period of development, the basally located cells of the mammary buds originating from the epidermis do not express smooth muscle markers, and the future myoepithelial cells gradually acquire features characteristic of smooth muscle differentiation during the postnatal mammary gland development [1]. Acquisition of the differentiated phenotype is accompanied by changes in adhesion systems (i.e. upregulation of specific integrin and laminin variant expression) [1].

In the human foetal breast, the first smooth muscle marker ( $\alpha$-actin) was found in basally located cells after 23 weeks of gestation [2]. In the adult mammary gland, the smooth muscle marker expression of myoepithelial cells appears to be heterogeneous. In the quiescent human breast, heavy caldesmon (an important regulator of the contractile function) is present in the myoepithelial cells of large ducts and galactophorous sinuses, but is not found in intralobular small ducts and acini [3]. Similarly, in the lactating rat mammary gland, ductal and alveolar myoepithelial cells display different patterns of expression of contractile, cytoskeletal and ECM smooth muscle markers [1]. This heterogeneity may reflect differences in functional properties and the diverse origins of the myoepithelial cells residing in the various parts of the mammary tree.

Previous observations resulting from studies performed with the mouse mammary gland have provided convincing evidence that the cap cells of the terminal end buds (TEB) can give rise to ductal myoepithelial cells [4]. The TEB are bulbous structures found in pubertal animals. They are located at the endings of mammary ducts advancing into the fat pad, and the cap cells form a basally located monolayer at their tip. Cap cells themselves do not express smooth muscle markers but, as the duct grows into the stroma, they move to the more proximal part of the duct and differentiate into myoepithelial cells. Although the TEB are present only in rapidly growing pubertal mammary glands, cell populations similar to cap cells may exist at the extremities of all growing buds at various developmental stages. Cap cells are unlikely to be the only source of myoepithelial progenitors, however, as experiments involving the serial transplantation of mammary tissue fragments have shown that precursor cells are distributed throughout the mammary tree rather than concentrated at any particular site [5] (reviewed in [6]).

Several data suggest the existence of bipotent mammary cell precursors that can give rise to both luminal and myoepithelial cells. Stingl et al. attempted to characterise the epithelial progenitor populations present in normal adult human mammary tissue by a combination of flow cytometry and in vivo colony formation assays [7]. The markers used to identify cells belonging to the two major mammary cell lineages (luminal and basal) included cytokeratins 8/18 and 19, EpCAM and MUC1 for luminal cells, and K14, high levels of $\alpha 6$-integrin and low levels of MUC1 for myoepithelial cells. This study by Stingl et al. suggested the presence of fate-restricted myoepithelial and luminal precursors, as well as bipotent progenitors. However, the authors did not analyse the expression of myoepithelium-specific contractile and cytoskeletal proteins, such as smooth muscle $\alpha$-actin. Furthermore, Lakhani et al.'s demonstration of identical genetic changes (loss of heterozygosity) in luminal and myoepithelial cells [8] provides convincing evidence in favour of the existence of a common precursor.

The precise location and molecular characteristics of myoepithelial precursor cells, with the exception of cap cells, are unknown. The results of experiments performed with cultured cells from human and mouse mammary glands suggest that myoepithelial cells may be derived from precursors within the luminal epithelial layer [9-13]. On the contrary, a recent study by Böcker et al. performed with human breast specimens describes a K5-positive cell population that might be a precursor of both luminal cells ('glandular epithelial') and myoepithelial cells [14]. Furthermore, Gudjonsson et al. isolated a mammary cell population with suprabasal characteristics (i.e. possessing luminal properties but negative for sialomucin, an apical marker of luminal cells) from human breast tissue [15]. These cells were used to establish a cell line displaying progenitor properties: the cells were able, in two-dimensional culture, to form branching structures resembling the terminal duct lobular units of the breast and containing basal and luminal cells. The authors suggested that in vivo, consistent with the characteristics exhibited in culture, these bipotent progenitor cells were located suprabasally and resided on myoepithelial cells.

Overall, the location and molecular markers of mammary progenitor cells, as well as the mechanisms controlling the maintenance of stem cells as such and their commitment to one of the two major mammary epithelial lineages, remain to be elucidated. 


\section{The myoepithelial cell layer harbours important regulatory molecules}

Numerous molecules implicated in important regulatory processes in other tissues are expressed differently in the two epithelial layers of the mammary gland. Several members of the epidermal growth factor family have been shown to play an important regulatory role in mammary gland development and in the differentiation of mammary epithelium (reviewed in [16]). In the mammary epithelium, the epidermal growth factor receptor expression level found in the cap cells and myoepithelial cells is significantly greater than that in the luminal cells $[17,18]$. This distribution, together with the results of studies carried out in culture with a mammary epithelial cell line displaying basal characteristics [19], suggests a specific role of epidermal growth factor receptor signalling in the control of the basal myoepithelial cell phenotype.

Gomm et al. have analysed the expression of basic fibroblast growth factor (FGF2) and fibroblast growth factor receptor 1 in luminal and myoepithelial cells isolated from the normal human breast [20]. The mRNA for FGF2 was found only in myoepithelial cells, whereas fibroblast growth factor receptor 1 , as estimated by immunolabelling, was present in luminal cells and, to a lesser extent, in myoepithelial cells. These results suggest that myoepithelial cell-derived FGF2 may be an important paracrine factor controlling luminal epithelial cell growth.

The Tcf transcription factors are central components of the $\mathrm{Wnt} / \beta$-catenin signalling pathway, which has been implicated in many aspects of development and tumourigenesis (reviewed in [21]). The members of the Tcf family exhibit differential tissue expression patterns. Tcf 4 and Tcf1 have been shown to be present in the mammary epithelium, with nuclear Tcf1 detected specifically in the basal mammary epithelial cells. The most abundant form of Tcf1 lacks the $\beta$-catenin binding domain and is therefore likely to act as a negative regulator of Wnt signalling. Consistent with this notion, mice lacking Tcf1 develop mammary adenomas [22]. These findings suggest a potential role for the Wnt signalling pathway in the control of basal mammary cell growth. Different members of the Wnt family have been found in the various mammary gland compartments. In particular, Wnt2 is apparently expressed by myoepithelial cells rather than luminal cells [23].

Activins belong to the transforming growth factor beta superfamily, many members of which are important regulators of differentiation and development, particularly for various smooth muscle and epithelial cell types. Human mammary myoepithelial cells in primary culture have been found to express both activin $\beta A$ and activin type II receptor, whereas other breast cell types do not [24]. The targeted expression of transforming growth factor beta in luminal cells results in the inhibition of mammary develop- ment [25]. To our knowledge, however, the involvement of the transforming growth factor beta signalling pathway in the differentiation of mammary myoepithelial cells has not yet been studied.

The most recently discovered members of the p53 family are p63 and p73. These transcription factors play a central role in the control of cell growth and survival, and they act as tumour suppressors. Immunohistochemical studies have shown that, in the mammary epithelium, both p63 and p73 are restricted to the myoepithelial cell layer [26-29]. A recently described human mammary epithelial cell line with basal properties has been shown to express a p63 variant, $\Delta N-p 63-\alpha$ [27]. The specific location of $p 63$ in a subset of basal mammary cells [27] is particularly intriguing because, in the epidermis, this p53 homologue identifies keratinocyte stem cells [30]. Moreover, p63 expression is critical for the maintenance of the progenitor cell population necessary for development and morphogenesis. All squamous epithelia and their derivatives, including the mammary gland, have been shown to be absent in p63-deficient mice [31,32]. Additional studies are required to elucidate the role of $p 63$ in mammary development.

\section{Perturbation of the myoepithelial cell-specific protein expression pattern}

There is a growing body of evidence to suggest that interference with the normal pattern of expression of genes encoding molecules specific to the myoepithelium, by ablation or overexpression, perturbs the growth and differentiation of the entire mammary epithelium. Several examples are now described.

P-cadherin is restricted to the basal layer of stratified and pseudostratified epithelia, and it is present only in the myoepithelial cells in the mammary gland. P-cadherin-deficient virgin mice display precocious mammary gland development [33]. They contain alveolus-like buds similar morphologically to those observed in early pregnant animals, with luminal cells producing milk proteins (caseins). This suggests that perturbation of the pattern of gene expression in myoepithelial cells may affect the growth and differentiation of luminal cells.

Parathyroid hormone-related protein (PTHrP) is implicated in a wide variety of biological processes during embryonic development and in adults. In the mammary gland, it is produced by both luminal and myoepithelial cells. However, only myoepithelial cells are responsive to PTHrP. The targeted overexpression of PTHrP in myoepithelial cells under the control of the $K 14$ gene promoter results in mammary hypoplasia characterised by deficient ductal branching in virgin mice, due to the high rates of apoptosis and the low rates of proliferation observed in TEB $[34,35]$. These findings provide further evidence that 
myoepithelial cells can participate in the control of growth, differentiation and morphogenetic events involving luminal cells, thereby demonstrating the contribution of these cells to normal mammary gland development.

Epithelial basal cell-specific promoters, such as those of the $K 5$ and $K 14$ genes, are often used to create transgenic mice for studies of developmental processes in the epidermis and hair follicles. Unfortunately, with few exceptions, the mammary phenotypes of mice bearing transgenes under the control of $K 5$ or $K 14$ promoters have not been analysed. In addition to the results obtained with the K14-PTHrP transgenic mouse line described earlier, a recently published study illustrates that basal keratin promoters can be used to address important questions concerning the mammary gland. Jonkers et al. showed that mice carrying conditional Brca2 and Trp53 alleles and a cre transgene under the control of the $\mathrm{K} 14$ gene promoter developed mammary tumours with luminal and myoepithelial characteristics [36]. It is currently unknown whether these tumours develop due to inactivation of the Brca2 and Trp53 genes in differentiated myoepithelial cells, or whether they result from the ablation of these genes in some K14-expressing early mammary precursor cells, as the $K 14$ promoter is active early in embryonic development.

Several members of the family of ephrin receptor tyrosine kinases and their ligands have been implicated in the regulation of pattern formation during embryogenesis. In the normal mouse mammary gland, the ephrin receptor EphB4 is found predominantly in myoepithelial cells, whereas expression of its ligand (ephrin-B2) is restricted to luminal cells [37]. In the rapidly growing tumours found in Wap-ras transgenic mice, the receptor ceased to be expressed on myoepithelial cells and was instead detected in the tumour cells [38]. The targeted overexpression of EphB4 in luminal mammary cells under the control of the mouse mammary tumour virus promoter affected the mammary gland development. The transgenic glands exhibited, on the one hand, high rates of apoptosis in pregnancy and, on the other, abnormal proliferation during the early stages of involution. Double-transgenic animals expressing the EphB4 and neuT genes developed mammary tumours earlier than did those expressing neuT only. Moreover, lung metastases were observed exclusively in the double-transgenic mice.

These data are an example of how the introduction of a myoepithelial cell-specific molecule into luminal cells results in the perturbation of the mammary epithelial cell response to physiological signals that normally induce proliferation, apoptosis or survival.

\section{Myoepithelial cells play a key role in the establishment of the mammary bilayer}

The integrity of the mammary epithelium is maintained by several distinct adhesion systems. The adhesive struc- tures involved in cell-cell contacts at the lateral surfaces of the luminal cells and between the luminal and the basal myoepithelial cells include desmosomes and cadherinmediated junctions. In contrast, hemidesmosomes and dense plaques specific to myoepithelial cells are localised to the sites of cell-ECM interactions. Within the cell, desmosomes and hemidesmosomes are associated with the intermediate filaments, whereas integrin-containing and cadherin-containing junctions are connected to the actin cytoskeleton, and are often referred to as adherens junctions. In accordance with the specific functions of basal myoepithelial cells in adhesion of the mammary epithelial bilayer to the basement membrane, integrins and cytoplasmic components of the cell-ECM adherens junctions (such as vinculin, $\alpha$-actinin, focal adhesion kinase and talin) are much more abundant in myoepithelial cells than in luminal epithelial cells [39].

Mammary myoepithelial cells may be expected to participate in ECM turnover, either permanently or during particular stages of mammary development, such as intensive growth in puberty or gland remodelling during involution. Thus, although most of the ECM-degrading enzymes found in the mammary gland are considered of stromal origin, myoepithelial cells produce several specific or ubiquitous matrix-degrading enzymes, in addition to numerous protease inhibitors (reviewed in [40]). A recently described angiogenesis-related matrix metalloproteinase, MMP19, was expressed by normal breast myoepithelial cells [41].

Gudjonsson et al. suggested that myoepithelial cells might play an essential role in the control of polarity in the bilayered mammary epithelium [42]. Indeed, luminal epithelial cells cultured in collagen-I gel formed acinus-like structures with reversed polarity, with apical markers expressed on their external surface and with basal markers expressed on the luminal side. The addition of myoepithelial cells led to the formation of acinus-like aggregates with the correct polarity. The mammary basement membrane component laminin 1 may replace myoepithelial cells in their instructive function, as in the reconstituted basement membrane material (Matrigel), even in the absence of myoepithelial cells, the polarity of the aggregates formed by luminal cells was correct. Myoepithelial cells isolated from breast tumours were not able to produce laminin 1 and, consistently, could not correct the polarity of the aggregates formed by luminal cells in collagen gel. These findings stress the importance of cell-ECM interactions in the establishment of basoapical polarity in the mammary epithelium, and illustrate the critical involvement of the myoepithelial cell as a source of the basement membrane material.

Desmosomes and cadherin-mediated adherens junctions are involved in the adhesion of the two mammary epithelium layers. The luminal cells express larger amounts of 
E-cadherin than do the myoepithelial cells, whereas $\mathrm{P}$-cadherin is expressed exclusively by the myoepithelial cells. This differential cadherin distribution may contribute to the segregation of the two major mammary cell types.

In addition, a recent elegant study revealed the important role played by desmosome components in the positioning of the luminal and myoepithelial cells in the mammary bilayer [43]. In this work, Runswick et al. investigated the role of desmosomes in the mammary epithelium organisation, using blocking peptides corresponding to the cell adhesion recognition sites of desmosomal cadherins. Incubation of mammary epithelial cells with a mixture of cell adhesion recognition peptides inhibiting the cadherins in the mammary epithelium prevented alveolar morphogenesis and demonstrated that desmosomes are absolutely required for the formation of multicellular branching structures. The desmosomal cadherins, desmocolin and desmoglein, are differentially expressed in the two mammary epithelial cell layers: Dsc2 and Dsg2 are present in both cell types, whereas Dsc3 and Dsg3 are restricted to the myoepithelium. In rotary culture, human breast epithelial cells can associate to form clusters resembling mammary alveoli, with centrally located luminal cells surrounded by an external ring of myoepithelial cells. The addition of cell adhesion recognition peptides corresponding to the myoepithelial cell-specific desmosomal cadherins, desmocolin Dsc3 and desmoglein Dsg3, interfered with this cell type-specific positioning. These results led Runswick et al. to conclude that the cell-cell adhesion might have a more dominant effect than cell-matrix interactions in cell positioning in the mammary bilayer [43].

\section{Mammary tumours with basal cell characteristics}

Most breast carcinomas express phenotypic markers suggestive of a luminal origin. In contrast, breast myoepitheliomas are rather rare. The basal keratins $\mathrm{K} 14$ and K17 have, however, been reported to be present in an important subset (20-33\%) of invasion breast carcinomas (reviewed recently in [44]). Moreover, analysis of the pattern of gene expression using complementary DNA microarrays has revealed a distinct tumour subclass accounting for $15 \%$ of all analysed tumours. In this subclass, expression levels were high for basal keratin genes and for other genes characteristic of basal mammary cells, such as those encoding the $\alpha 3$ and $\gamma 2$ laminin chains and the $\beta 4$ integrin subunit [45].

Further analysis of this basal cell-like tumour subclass has shown that TP53 was mutated in $82 \%$ of the samples analysed, whereas only $13 \%$ of the tumours in the luminal subclass contained mutated TP53 [46]. Previous studies have indicated that mutations in TP53 are associated with a poor prognosis and a poor response to systemic therapy consistently found to be associated with short survival time. Expression of the entire set of basal cell markers by this subclass of breast tumours indicates a possible origin from a mammary cell progenitor with molecular characteristics of basal cells.

\section{Tumour suppressor potential of myoepithelial cells}

Many lines of evidence suggest that differentiated myoepithelial cells are 'natural tumour suppressors' [47] (for reviews, see $[48,49]$ ) because they inhibit proliferation in breast carcinoma cells by inducing growth arrest and apoptosis, because they interfere with the invasive behaviour of tumour cells and because they inhibit angiogenesis. Indeed, mammary myoepithelial cells and cell lines obtained from benign myoepithelial tumours produce relatively high levels of protease inhibitors and active antiangiogenic factors, such as protease nexin II, $\alpha 1$-antitrypsin, a $31 \mathrm{kDa}$ serine protease inhibitor, tissue inhibitor of metalloproteinase 1 , thrombospondin-1 and the soluble basic fibroblast growth factor receptor [47,50-52].

Maspin, a member of the serpin family of serine proteases, was identified by subtractive hybridisation of cDNAs from normal versus tumourigenic human mammary epithelial cells [53]. This serpin, produced by myoepithelial cells, functions as a tumour suppressor and can inhibit metastasis in vivo (reviewed in [54]). A new myoepitheliumspecific serine proteinase inhibitor was recently identified and described by Xiao et al. [55]. This molecule, when expressed in human breast cancer cells, abolished their growth, decreased their invasive potential and prevented tumour dissemination in vivo. In addition to producing these anti-invasive and anti-angiogenic molecules, myoepithelial cells have also been shown to possess CD44 shedding activity and to produce soluble CD44, which blocks the adhesion and migration of human carcinoma cells on hyaluronic acid-coated surfaces $[56,57]$.

Finally, the analysis of myoepithelial marker expression remains a commonly used approach to distinguish between benign and malignant tumours, or to detect stromal invasion (reviewed recently in [58]).

\section{Conclusions}

The mammary myoepithelial cells, due to their specific differentiation programme, are able to integrate multiple signals from the neighbouring cells, from the underlying basement membrane and from the connective tissue. In turn, these cells have a major impact on luminal cell growth and differentiation, and they play a key role in the establishment and the maintenance of the mammary epithelium architecture. However, we currently know very little about the cellular and molecular characteristics of myoepithelial cell precursors, as well as the unique mechanisms that control the myoepithelial cell phenotype and 
allow the expression of its double (smooth muscle and epithelial) identity. Moreover, the nature of the signals involved in communication between luminal and basal myoepithelial cells is largely unknown. These unresolved questions provide a vast area for future investigation.

\section{Acknowledgements}

MAD is Chargé de Recherche and MAG is Directeur de Recherche at the Institut National de la Santé et de la Recherche Médicale. MMF was supported by a fellowship from the Institut Curie and the Fondation pour la Recherche Médicale. The authors thank the Association pour la Recherche contre le Cancer (ARC 4440) for research funding.

\section{References}

1. Deugnier MA, Moiseyeva EP, Thiery JP, Glukhova M: Myoepithelial cell differentiation in the developing mammary gland: progressive acquisition of smooth muscle phenotype. Dev Dyn 1995, 204:107-117.

2. Anbazhagan R, Osin PP, Bartkova J, Nathan B, Lane EB, Gusterson BA: The development of epithelial phenotypes in the human fetal and infant breast. J Pathol 1998, 184:197-206.

3. Lazard D, Sastre X, Frid MG, Glukhova MA, Thiery JP, Koteliansky VE: Expression of smooth muscle-specific proteins in myoepithelium and stromal myofibroblasts of normal and malignant human breast tissue. Proc Natl Acad Sci USA 1993, 90:9991003.

4. Williams JM, Daniel CW: Mammary ductal elongation: differentiation of myoepithelium and basal lamina during branching morphogenesis. Dev Biol 1983, 97:274-290.

5. Smith GH, Medina D: A morphologically distinct candidate for an epithelial stem cell in mouse mammary gland. J Cell Sci 1988, 90:173-183.

6. Smith GH, Chepko G: Mammary epithelial stem cells. Microsc Res Tech 2001, 52:190-203.

7. Stingl J, Eaves CJ, Zandieh I, Emerman JT: Characterization of bipotent mammary epithelial progenitor cells in normal adult human breast tissue. Breast Cancer Res Treat 2001, 67:93109.

8. Lakhani SR, Chaggar R, Davies S, Jones C, Collins N, Odel C, Stratton MR, O'Hare MJ: Genetic alterations in 'normal' luminal and myoepithelial cells of the breast. J Pathol 1999, 189:496503.

9. Joshi K, Smith JA, Perusinghe N, Monoghan P: Cell proliferation in the human mammary epithelium. Differential contribution by epithelial and myoepithelial cells. Am J Pathol 1986, 124: 199-206.

10. Kao CY, Nomata K, Oakley CS, Welsch CW, Chang CC: Two types of normal human breast epithelial cells derived from reduction mammoplasty: phenotypic characterization and response to SV40 transfection. Carcinogenesis 1995, 16:531538.

11. Kang KS, Morita I, Cruz A, Jeon YJ, Trosko JE, Chang CC: Expression of estrogen receptors in a normal human breast epithelial cell type with luminal and stem cell characteristics and its neoplastically transformed cell lines. Carcinogenesis 1997, 18:251-257.

12. Smalley MJ, Titley J, Paterson H, Perusinghe N, Clarke C, O'Hare $\mathrm{MJ}$ : Differentiation of separated mouse mammary luminal epithelial and myoepithelial cells cultured on EHS matrix analyzed by indirect immunofluorescence of cytoskeletal antigens. J Histochem Cytochem 1999, 47:1513-1524.

13. Pechoux C, Gudjonsson T, Ronnov-Jessen L, Bissell MJ, Petersen OW: Human mammary luminal epithelial cells contain progenitors to myoepithelial cells. Dev Biol 1999, 206:88-99.

14. Böcker W, Moll R, Poremba $C$, Holland R, Van Diest PJ, Dervan P, Burger H, Wai D, Ina Diallo R, Brandt B, Herbst H, Schmidt A, Lerch $\mathrm{MM}$, Buchwallow IB: Common adult stem cells in the human breast give rise to glandular and myoepithelial cell lineages: a new cell biological concept. Lab Invest 2002, 82:737-746.

15. Gudjonsson T, Villadsen R, Nielsen HL, Ronnov-Jessen L, Bissell MJ, Petersen OW: Isolation, immortalization, and characterization of a human breast epithelial cell line with stem cell properties. Genes Dev 2002, 16:693-706.
16. Olayioye MA, Neve RM, Lane HA, Hynes NE: The ErbB signaling network: receptor heterodimerization in development and cancer. Embo J 2000, 19:3159-3167.

17. Coleman S, Silberstein GB, Daniel CW: Ductal morphogenesis in the mouse mammary gland: evidence supporting a role for epidermal growth factor. Dev Biol 1988, 127:304-315.

18. Moller P, Mechtersheimer G, Kaufmann M, Moldenhauer G, Momburg F, Mattfeldt T, Otto HF: Expression of epidermal growth factor receptor in benign and malignant primary tumours of the breast. Virchows Arch A Pathol Anat Histopathol 1989, 414:157-164.

19. Deugnier MA, Faraldo MM, Rousselle P, Thiery JP, Glukhova MA: Cell-extracellular matrix interactions and EGF are important regulators of the basal mammary epithelial cell phenotype. $J$ Cell Sci 1999, 112:1035-1044.

20. Gomm JJ, Browne PJ, Coope RC, Bansal GS, Yiangou C, Johnston $\mathrm{CL}$, Mason R, Coombes RC: A paracrine role for myoepithelial cell-derived FGF2 in the normal human breast. Exp Cell Res 1997, 234:165-173.

21. Wodarz A, Nusse R: Mechanisms of Wnt signaling in development. Annu Rev Cell Dev Biol 1998, 14:59-88.

22. Roose J, Huls G, van Beest M, Moerer P, van der Horn K, Goldschmeding R, Logtenberg T, Clevers $\mathrm{H}$ : Synergy between tumor suppressor APC and the beta-catenin-Tcf4 target Tcf1. Science 1999, 285:1923-1926.

23. Buhler TA, Dale TC, Kieback C, Humphreys RC, Rosen JM: Localization and quantification of Wnt-2 gene expression in mouse mammary development. Dev Biol 1993, 155:87-96.

24. Liu QY, Niranjan B, Gomes P, Gomm JJ, Davies D, Coombes RC Buluwela L: Inhibitory effects of activin on the growth and morphogenesis of primary and transformed mammary epithelial cells. Cancer Res 1996, 56:1155-1163.

25. Pierce DF Jr, Johnson MD, Matsui Y, Robinson SD, Gold LI, Purchio AF, Daniel CW, Hogan BL, Moses HL: Inhibition of mammary duct development but not alveolar outgrowth during pregnancy in transgenic mice expressing active TGFbeta 1. Genes Dev 1993, 7:2308-2317.

26. Barbareschi M, Pecciarini L, Cangi MG, Macri E, Rizzo A, Viale G, Doglioni C: p63, a p53 homologue, is a selective nuclear marker of myoepithelial cells of the human breast. Am J Surg Pathol 2001, 25:1054-1060.

27. DiRenzo J, Signoretti S, Nakamura N, Rivera-Gonzalez R, Sellers W, Loda M, Brown M: Growth factor requirements and basal phenotype of an immortalized mammary epithelial cell line. Cancer Res 2002, 62:89-98.

28. Di Como CJ, Urist MJ, Babayan I, Drobnjak M, Hedvat CV, TeruyaFeldstein J, Pohar K, Hoos A, Cordon-Cardo C: p63 expression profiles in human normal and tumor tissues. Clin Cancer Res 2002, 8:494-501.

29. Yamamoto T, Oda K, Miyazaki K, Ichigotani Y, Takenouchi $Y$, Kamei T, Shirafuji N, Nimura Y, Hamaguchi M, Matsuda S: p73 is highly expressed in myoepithelial cells and in carcinomas with metaplasia. Int J Oncol 2001, 19:271-276.

30. Pellegrini G, Dellambra E, Golisano O, Martinelli E, Fantozzi I, Bondanza S, Ponzin D, McKeon F, De Luca M: p63 identifies keratinocyte stem cells. Proc Natl Acad Sci USA 2001, 98: 3156-3161.

31. Mills $A A$, Zheng $B$, Wang $X J$, Vogel $H$, Roop DR, Bradley A: p63 is a p53 homologue required for limb and epidermal morphogenesis. Nature 1999, 398:708-713.

32. Yang A, Schweitzer R, Sun D, Kaghad M, Walker N, Bronson RT, Tabin C, Sharpe A, Caput D, Crum C, McKeon F: p63 is essential for regenerative proliferation in $\operatorname{limb}$, craniofacial and epithelial development. Nature 1999, 398:714-718.

33. Radice GL, Ferreira-Cornwell MC, Robinson SD, Rayburn H, Chodosh LA, Takeichi M, Hynes RO: Precocious mammary gland development in P-cadherin-deficient mice. J Cell Biol 1997, 139:1025-1032.

34. Wysolmerski JJ, McCaughern-Carucci JF, Daifotis AG, Broadus $A E$, Philbrick WM: Overexpression of parathyroid hormonerelated protein or parathyroid hormone in transgenic mice impairs branching morphogenesis during mammary gland development. Development 1995, 121:3539-3547.

35. Dunbar ME, Dann P, Brown CW, Van Houton J, Dreyer B, Philbrick WP, Wysolmerski JJ: Temporally regulated overexpression of parathyroid hormone-related protein in the mammary gland reveals distinct fetal and pubertal phenotypes. J Endocrinol 2001, 171:403-416. 
36. Jonkers J, Meuwissen R, van der Gulden H, Peterse H, van der Valk M, Berns A: Synergistic tumor suppressor activity of BRCA2 and p53 in a conditional mouse model for breast cancer. Nat Genet 2001, 29:418-425.

37. Nikolova Z, Djonov V, Zuercher G, Andres AC, Ziemiecki A: Celltype specific and estrogen dependent expression of the receptor tyrosine kinase EphB4 and its ligand ephrin-B2 during mammary gland morphogenesis. J Cell Sci 1998, 111: 2741-2751.

38. Munarini N, Jager R, Abderhalden S, Zuercher G, Rohrbach V, Loercher S, Pfanner-Meyer B, Andres AC, Ziemiecki A: Altered mammary epithelial development, pattern formation and involution in transgenic mice expressing the EphB4 receptor tyrosine kinase. J Cell Sci 2002, 115:25-37.

39. Glukhova M, Koteliansky V, Sastre X, Thiery JP: Adhesion systems in normal breast and in invasive breast carcinoma. Am J Pathol 1995, 146:706-716.

40. Rudolph-Owen LA, Matrisian LM: Matrix metalloproteinases in remodeling of the normal and neoplastic mammary gland. J Mammary Gland Biol Neoplasia 1998, 3:177-189.

41. Djonov V, Hogger K, Sedlacek R, Laissue J, Draeger A: MMP-19: cellular localization of a novel metalloproteinase within normal breast tissue and mammary gland tumours. J Pathol 2001, 195:147-155.

42. Gudjonsson T, Ronnov-Jessen L, Villadsen R, Rank F, Bissell MJ, Petersen OW: Normal and tumor-derived myoepithelial cells differ in their ability to interact with luminal breast epithelial cells for polarity and basement membrane deposition. J Cell Sci 2002, 115:39-50.

43. Runswick SK, O'Hare MJ, Jones L, Streuli CH, Garrod DR: Desmosomal adhesion regulates epithelial morphogenesis and cell positioning. Nat Cell Biol 2001, 3:823-830.

44. Petersen OW, Lind Nielsen $H$, Gudjonsson T, Villadsen $R$ Ronnov-Jessen L, Bissell MJ: The plasticity of human breast carcinoma cells is more than epithelial to mesenchymal conversion. Breast Cancer Res 2001, 3:213-217.

45. Perou CM, Sorlie T, Eisen MB, van de Rijn M, Jeffrey SS, Rees CA, Pollack JR, Ross DT, Johnsen H, Akslen LA, Fluge O, Pergamenschikov A, Williams $C$, Zhu SX, Lonning PE, Borresen-Dale $A L$, Brown PO, Botstein D: Molecular portraits of human breast tumours. Nature 2000, 406:747-752.

46. Sorlie T, Perou CM, Tibshirani R, Aas T, Geisler S, Johnsen H, Hastie T, Eisen MB, van de Rijn M, Jeffrey SS, Thorsen T, Quist H, Matese JC, Brown PO, Botstein D, Eystein Lonning P, BorresenDale AL: Gene expression patterns of breast carcinomas distinguish tumor subclasses with clinical implications. Proc Natl Acad Sci USA 2001, 98:10869-10874.

47. Sternlicht MD, Kedeshian P, Shao ZM, Safarians S, Barsky SH: The human myoepithelial cell is a natural tumor suppressor. Clin Cancer Res 1997, 3:1949-1958.

48. Lakhani SR, O'Hare MJ: The mammary myoepithelial cell Cinderella or ugly sister? Breast Cancer Res 2001, 3:1-4.

49. Sternlicht MD, Barsky SH: The myoepithelial defense: a host defense against cancer. Med Hypotheses 1997, 48:37-46.

50. Nguyen M, Lee MC, Wang JL, Tomlinson JS, Shao ZM, Alpaugh ML, Barsky SH: The human myoepithelial cell displays a multifaceted anti-angiogenic phenotype. Oncogene 2000, 19:34493459.

51. Shao ZM, Nguyen M, Alpaugh ML, O'Connell JT, Barsky SH: The human myoepithelial cell exerts antiproliferative effects on breast carcinoma cells characterized by p21WAF1/CIP1 induction, G2/M arrest, and apoptosis. Exp Cell Res 1998, 241:394-403.

52. Shao ZM, Radziszewski WJ, Barsky SH: Tamoxifen enhances myoepithelial cell suppression of human breast carcinoma progression in vitro by two different effector mechanisms. Cancer Lett 2000, 157:133-144.

53. Zou Z, Anisowicz A, Hendrix MJ, Thor A, Neveu M, Sheng S, Rafidi K, Seftor E, Sager R: Maspin, a serpin with tumor-suppressing activity in human mammary epithelial cells. Science 1994, 263:526-529.

54. Streuli $\mathrm{CH}$ : Maspin is a tumour suppressor that inhibits breast cancer tumour metastasis in vivo. Breast Cancer Res 2002, 4: 137-140.

55. Xiao G, Liu YE, Gentz R, Sang QA, Ni J, Goldberg ID, Shi YE: Suppression of breast cancer growth and metastasis by a serpin myoepithelium-derived serine proteinase inhibitor expressed in the mammary myoepithelial cells. Proc Nat/ Acad Sci USA 1999, 96:3700-3705.

56. Lee MC, Alpaugh ML, Nguyen M, Deato M, Dishakjian L, Barsky $\mathrm{SH}$ : Myoepithelial-specific CD44 shedding is mediated by a putative chymotrypsin-like sheddase. Biochem Biophys Res Commun 2000, 279:116-123.

57. Alpaugh ML, Lee MC, Nguyen M, Deato M, Dishakjian L, Barsky $\mathrm{SH}$ : Myoepithelial-specific CD44 shedding contributes to the anti-invasive and antiangiogenic phenotype of myoepithelial cells. Exp Cell Res 2000, 261:150-158.

58. Yaziji H, Gown AM, Sneige N: Detection of stromal invasion in breast cancer: the myoepithelial markers. Adv Anat Pathol 2000, 7:100-109. 